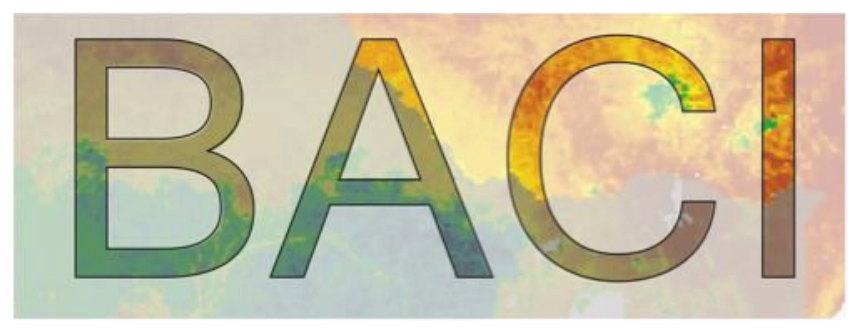

\title{
Improved tree-ring archives will support earth-system science
}

\section{Postprint version}

Flurin Babst, Benjamin Poulter, Paul Bodesheim, Miguel D. Mahecha and David C. Frank

\section{Published in: $\quad$ Nature Ecology \& Evolution}

Reference: Babst, F., Poulter, B., Bodesheim, P., Mahecha, M. D., \& Frank, D. C. (2017). Improved tree-ring archives will support earth-system science (commentary). Nature Ecology \& Evolution, 1:0008. doi:10.1038/s41559-016-0008.

Web link: https://www.nature.com/articles/s41559-016-0008 


\title{
Improved tree-ring archives will support earth-system science
}

\author{
Flurin Babst, Benjamin Poulter, Paul Bodesheim, Miguel D. Mahecha and David C. Frank
}

A steep decline in archiving could make large tree-ring datasets irrelevant. But increased spatiotemporal
coverage, the addition of novel parameters at sub-annual resolution, and integration with other in situ and
remote Earth observations will elevate tree-ring data as an essential component of global-change research.

$\mathrm{M}$ ost tree species in seasonal climates undergo a regular dormancy that results in annual growth increments. These tree rings can be used to derive millennial-length records of variability in forest growth; such records have been highlighted in reports from the Intergovernmental Panel on Climate Change (IPCC) as a key resource in reconstructions of climate in pre-instrumental eras. Treering data are also increasingly being used to explore the question of how forests will respond to future environmental conditions. This has involved space-for-time forecasting of climate-induced growth changes ${ }^{1}$, tracking biome shifts ${ }^{2}$, assessing legacies of climate extremes $^{3}$, quantifying tree physiological responses to climate ${ }^{4}$, benchmarking mechanistic models ${ }^{5}$

and exploring the basic mechanisms of tree growth through sub-annual tree-ring studies ${ }^{6}$.

Large public archives make it feasible to tackle such topics across a broad range of environments. The International Tree-Ring Data Bank (ITRDB), which is managed by the US National Oceanic and Atmospheric Administration, is the largest global treering database. It currently lists more than 850 contributors and contains data from 4,200 sites on 6 continents. The parameter of total ring width is available at nearly all sites, and additional parameters are available for some sites, including earlywood and latewood width (at 614 and 616 sites, respectively), maximum wood density (581 sites) and stable-isotope measurements (24 sites). The genera Pinus, Picea and Quercus are most commonly represented, together constituting more than $50 \%$ of the database. The number of chronologies available for each year peaks in the midtwentieth century and plummets dramatically thereafter: at $44 \%$ of sites, the last measurement was before 1990 , at $77 \%$ of sites before 2000 and at $98 \%$ of sites before 2010 .
The negative consequences of this decline in data availability for palaeoclimatological research and the need to regularly update existing chronologies have already been noted $^{7}$. Here, we emphasize that the low coverage of tree-ring data from diverse geographic and climatic space over the past decade is limiting opportunities to integrate the global tree-ring network with newer observation platforms.

Indeed, the decrease in tree-ring data is in stark contrast with the increasing availability of spatially and temporally highly resolved data from satellite-retrieved Earth observations (EO; Fig. 1a). Large satellite-based initiatives established since the 1980s, such as those of NASA's Decadal Survey and the Climate Change Initiative

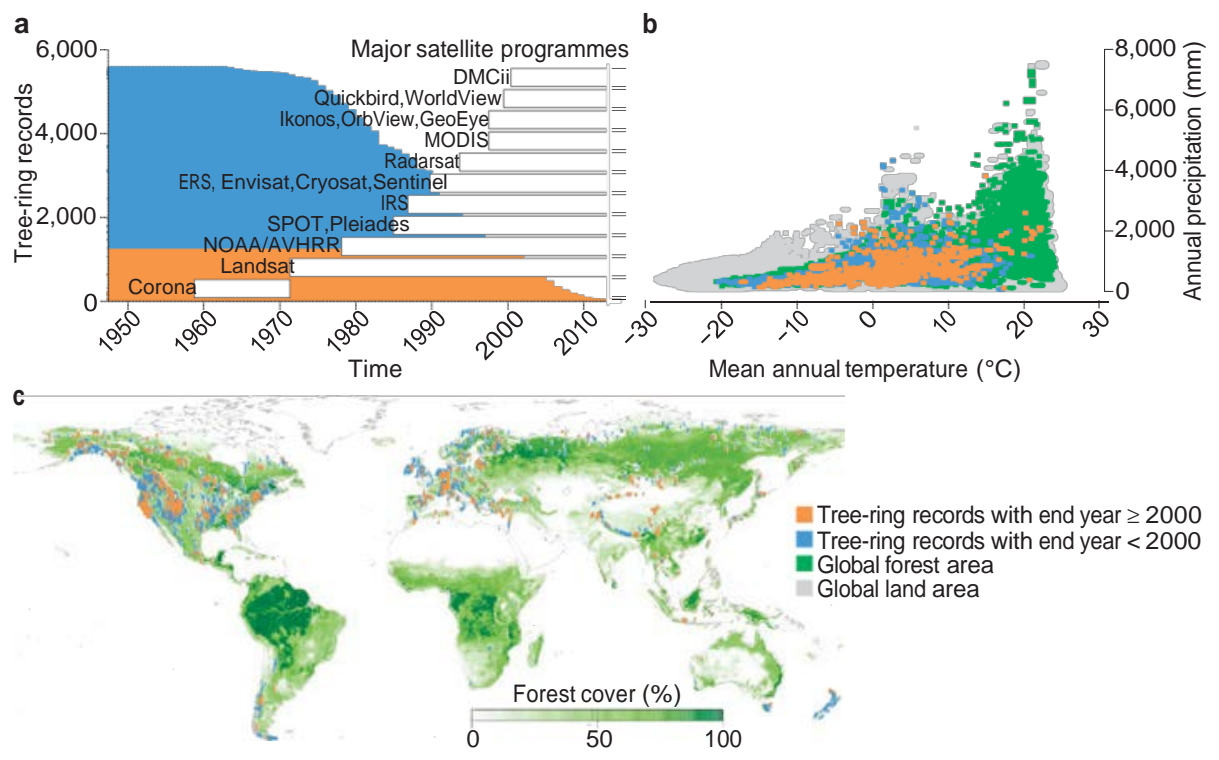

Figure 1 | The coverage of satellite and tree-ring records since 1950. a, Many tree-ring records from the International Tree-Ring Data Bank (ITRDB) end before data from major polar-orbiting satellite programmes began to become available. b,c, The reduction of ITRDB data since 2000 is seen in both climate (b) and geographic space (c). Global forest cover data was obtained from MODIS Land Cover and forests were defined where the fractional sum of forested categories was greater than $60 \%$ within a grid cell. 
EO data; (2) other in situ measurements related to forest growth, such as time-lapse photography of vegetation (phenocams) or eddy-covariance measurements of gas exchange between the biosphere and the atmosphere ${ }^{9}$; and (3) data on forest composition, management and tree dimensions recorded in national forest inventories (NFIs) ${ }^{10}$.

By integrating tree-ring data with EOs, NFIs and other in situ information, systematic coverage of forests - in terms of climate zones, species composition and forest demography - becomes a reachable goal. Combined analyses may also allow climate-induced variability in forest growth to be disentangled from that driven by community-level ecological processes. Unfortunately, however, tree-ring sampling is rudimentary or non-existent in most NFIs. And although the ITRDB has reasonable coverage of forests that occupy climate spaces with a mean annual temperature of less than $15^{\circ} \mathrm{C}$, its coverage of warmer forest regimes is patchy (Fig. 1b,c). Of further concern is the predominance of data from marginal growth environments, which probably reflects a collection bias stemming from the fact that climatic signals are more readily extracted in tree-ring records from such regions.

One numerical solution to fill in the gaps of existing tree-ring data is to use statistical upscaling with machine-learning techniques, whereby climate variables selected on the basis of observed climategrowth relationships are used to estimate radial tree growth in areas without existing records. Promising results have also been achieved with process-based modelling approaches to produce synthetic tree-ring records using relatively simple and globally available input parameters ${ }^{11}$. But global treering networks will always struggle to keep up with EOs in terms of spatiotemporal data acquisition. Hence, priorities need to be set regarding the locations and parameters of future research efforts. We recommend three areas for prioritization:

(1) Strengthen the systematic coverage of forest biomes. This must involve targeted sampling efforts and rapid contribution of new (or updated ${ }^{7}$ ) records to the ITRDB. We suspect that simply a more-open, datasharing culture could begin to address the unbalanced geographic distribution of ITRDB sites (Fig. 1c) and thereby already increase the use of tree-ring data for global research. Future field studies should focus on currently underrepresented ecosystems, especially to augment data coverage in warmer regions. Although the rhythm of growing and dormant seasons is not known in many tropical tree species, regular annual growth rings have been confirmed in 230 species $^{12}$, suggesting that dendrochronology possesses much unrealized potential in tropical biomes. Intensified international collaborations will be required to enhance sampling of these highly productive and rapidly diminishing forests, but establishing such collaborations should be easier than installing long-term research sites, which are also sparse in the tropics.

(2) Increase coordination with EO networks. Relevant networks include FLUXNET (a global network of eddy-covariance measurement sites), NEON (the US network of ecological monitoring sites), ICOS (the European network of greenhouse gas measuring stations), DendroGlobal (a developing network of dendrometer data) and long-term monitoring plots in NFIs. Routine tree-ring data collection at or near the research sites of these networks will bring benefits to multiple research communities, but requires representative samples of the respective forest stands. This is currently not the case for most sites on the ITRDB, where sampling has often targeted dominant trees from a vaguely defined area, and where key variables common to NFIs, such as stand density, tree dimensions, and forest demography are unknown. On-going efforts to facilitate and standardize the collection and archiving of such information ${ }^{13}$ will increase data compatibility, and enhance the ability to estimate broadly relevant parameters such as annual biomass increment.

(3) Promote measurements of parameters beyond radial growth increment. Continuous advances in quantitative wood anatomy ${ }^{6}$, stable-isotope analysis of wood compounds and digital imaging of wood surfaces are permitting increasingly rapid processing of tree-ring samples at sub-annual resolution. Such data complement information obtained through radial growth measurements and allow for refined comparisons with other temporally resolved in situ measurements. This may open research avenues in ecophysiology, structure-function trade-offs and climate reconstruction. Extending the list of parameters on the ITRDB is thus a collective wish that deserves allocation of the necessary resources.

If greenhouse gas emissions continue to follow their current trajectories, it is predicted that over one third of the global land surface will experience climatic conditions without historical analogue by $2100^{14}$. This warming will be accompanied by an unprecedented rise in atmospheric $\mathrm{CO}_{2}$ concentration, nitrogen deposition and disturbances - both natural and humaninduced. The structure of mechanistic models has been identified as the primary source of uncertainty in the context of projecting ecosystem states and changes ${ }^{15}$. Attempts to refine these models will greatly benefit from an integrated suite of empirical observations, including tree-ring, EO, and forest-monitoring datasets ${ }^{3-5,8,10,11}$. We expect that joint observational and computational approaches will continue to improve projections of climate impacts on forest ecosystems, but the necessary in situ data can only be developed and maintained through targeted community-wide efforts. $]$

Flurin Babst ${ }^{1,2}$, Benjamin Poulter ${ }^{3,4,5}$, Paul Bodesheim ${ }^{6}$, Miguel D. Mahecha $a^{6,7}$ and David C. Frank ${ }^{1,8}$ are at ${ }^{1}$ Dendroclimatology, Swiss Federal Research Institute WSL, Zürcherstrasse 111, CH-8903 Birmensdorf, Switzerland; ${ }^{2}$ W. Szafer Institute of Botany, Polish Academy of Sciences, ul. Lubicz 46, 31-512 Krakow, Poland; ' ${ }^{3}$ Department of Ecology, Montana State University, Bozeman, Montana, USA; ${ }^{4}$ NASA Goddard Space Flight Center, Greenbelt, Maryland 20771, USA; ${ }^{5}$ Department of Geographical Sciences, University of Maryland, College Park, Maryland 20742, USA; ${ }^{6}$ Max Planck Institute for Biogeochemistry, 07745 Jena, Germany; ${ }^{7}$ German Centre for Integrative Biodiversity Research (iDiv) Halle-Jena-Leipzig, 04103 Leipzig, Germany; and ${ }^{8}$ Laboratory of TreeRing Research, University of Arizona, 1215 E Lowell Street, Tucson, Arizona 85721, USA. e-mail: flurin.babst@wsl.ch

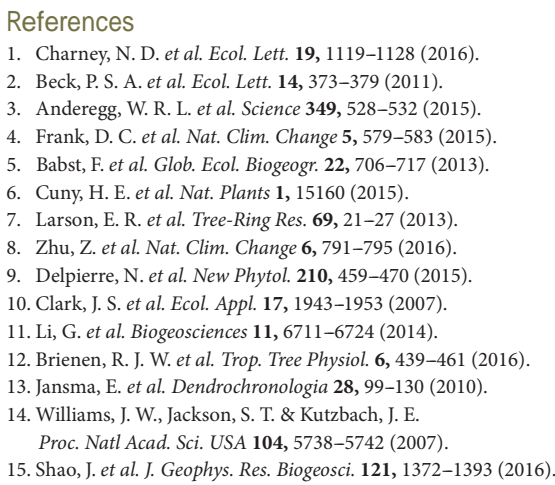

\section{Acknowledgements}

We acknowledge funding from the EU-H2020 program (grant 640176, 'Detecting changes in essential ecosystem and biodiversity properties - towards a Biosphere Atmosphere Change Index: BACI') and the Swiss National Science Foundation (\#P300P2_154543). We thank all ITRDB contributors, advisors and curators, and K. Seftigen, J. Björklund, A. Babst-Kostecka, M. E. K. Evans, and O. Bouriaud for fruitful discussions.

\section{Author contributions}

F.B. led the writing, homogenized the tree-ring data, and produced the figure, with critical input from D.C.F. Text contributions from B.P., P.B., and M.D.M. enriched the discussion around tree-ring integration with Earth observations, mechanistic models, and machine learning techniques. 